ИЗВЕСТИЯ АКАДЕМИИ НАУК ЭСТОНСКОИ ССР. ФИЗИКА * МАТЕМАТИКА

PROCEEDINGS OF THE ACADEMY OF SCIENCES OF THE ESTONIAN SSR.

PHYSICS * MATHEMATICS

1987, 36, 3

удК 514.75

М. ВЯЛЬЯС

\title{
ОБ ИЗОТЕРМИЧЕСКИХ ПОДМНОГООБРАЗИЯХ В КОНФОРМНО ПЛОСКОМ ПРОСТРАНСТВЕ
}

\author{
(Представил Г. Вайникко)
}

\section{§ 1. Введение и результаты}

1. Конформно плоские подмногообразия в конформно плоских римановых многообразиях привлекают внимание исследователей с начала $\mathrm{XX}$ века до наших дней. Основным отправным результатом является теорема Картана-Схоутена $\left[{ }^{1,2}\right]$, по которому гиперповерхность $V_{n}(n>3)$ в евклидовом пространстве $E_{n+1}$ является конформно плоской тогда и только тогда, когда ее вторая фундаментальная форма имеет собственное значение кратности $\geqslant n-1$, т. е. когда ее главные кривизны $k_{1}, \ldots, k_{n}$ обладают свойством $k_{1}=\ldots=k_{n-1}$. Такие гиперповерхности называются квазиомбилическими (см. $\left.\left[{ }^{3}\right]\right)$. В $\left[{ }^{4}\right]$ выяснено, что они представляют собой либо гиперсферы, либо огибающие однопараметрических семейств гиперсфер.

Конформно плоские подмногообразия $V_{n}$ коразмерности $m>1$ в конформно плоском римановом многообразии $V_{n+m}$ рассматривались в $\left[{ }^{5}\right]$, где для случая $n>3$ даны аналитические необходимые и достаточные условия, чтобы $V_{n}$ в таком $V_{n+m}$ было конформно плоским. В [ $\left.{ }^{6}\right]$ этот результат распространен на случай $n=3$.

Один интересный подкласс конформно плоских подмногообразий $V_{n}$ составляют вполне квазиомбилические подмногообразия $V_{n}(n>3)$ конформно плоского $V_{n+m}$ (см. $\left[{ }^{3}\right]$ гл. $\left.5 ;\left[{ }^{7}\right]\right)$. Подмногообразие $V_{n}$ риманова пространства $V_{n+m}$ называется вполне квазиомбилическим, если в некоторой окрестности каждой его точки существуют $m$ попарно ортогональных нормальных векторных полей, таких, что $V_{n}$ квазиомбилическое относительно каждого из них. Известно (см. $\left.\left[{ }^{3}\right]\right)$, что вполне квазиомбилическое подмногообразие $V_{n}(n>3)$ в конформно плоском $V_{n+m}$ является конформно плоским.

2. Теорема Қартана-Схоутена обобщена в $\left[{ }^{8}\right]$ на случай подмногообразия $V_{n}$ с плоской нормальной связностью (т. е. с сетью линий кривизны) при размерности $n>3$ и коразмерности $m>1$ в конформно плоском $V_{n+m}$. Главные кривизны гиперповерхности заменяются при этом главными векторами кривизны, т. е. векторами нормальной кривизны $\mathbf{k}_{1}, \ldots, \mathbf{k}_{n}$ линий кривизны рассматриваемого подмногообразия (cM. § 2 ).

Теорем а 1. (см. $\left.\left[{ }^{8}\right]\right)$. Пусть $V_{n}$ является подмногообразием размерности $n>3$ с плоской нормальной связностью в конформно пло- 
ском $V_{n+m}$. Тогда $V_{n}$ является конформно плоским тогда и только тогда, когда

$$
\left\langle\mathbf{k}_{p}-\mathbf{k}_{q}, \mathbf{k}_{r}-\mathbf{k}_{s}\right\rangle=0
$$

при различных значениях индексов $p, q, r$, s. B случае $m<n-2$ конформно плоское $V_{n}$, удовлетворяющцее этим условиям, является вполне квазиомбилическим.

Известно, что вектором нормальной кривизны в направлении $\mathbf{f}=\sum_{i=1}^{n} \varphi^{i} \mathbf{e}_{i}$, где $\mathbf{e}_{1}, \ldots, \mathbf{e}_{n}-$ единичные касательные векторы линий кривизны, является вектор $\mathrm{k}=\sum_{i=1}^{n} \mathrm{k}_{i}\left(\varphi^{i}\right)^{2}$. Его конец в точке $X \in V_{n}$ описывает индикатрису кривизны $\left[{ }^{9}\right]$, которая является выпуклой оболочкой множества точек $K_{1}, \ldots, K_{n}$ с радиус-векторами $\mathbf{k}_{1}, \ldots, \mathbf{k}_{n}$. Если нормальное пространство $T_{X} \perp\left(V_{n}\right)$ имеет размерность $m \geqslant n-1$ и точки $K_{1}, \ldots, K_{n}$ находятся в общем положении, тогда индикатриса кривизны является симплексом. Этот симплекс обозначим через $\Delta_{X}$.

Симплекс в евклидовом пространстве $E_{m}$ называется ортоцентрическим, если все его высоты пересекаются в одной точке, называемой ортоцентром. Для этого необходимо и достаточно, чтобы направления всех двух его неприлежащих ребер были ортогональны $\left[{ }^{10}\right]$. Частным случаем является прямоугольный симплекс, у которого существует одна вершина, называемая особой, причем исходящие из нее ребра попарно ортогональны; в этом случае орто-центром является эта особая вершина.

Аффинную оболочку точек $K_{1}, \ldots, K_{n}$ в пространстве $T \frac{\perp}{X}\left(V_{n}\right)$ обозначим через $A_{X}\left(K_{1}, \ldots, K_{n}\right)$, его размерность через $m_{1}$. Ясно, что $m_{1} \leqslant n-1$.

Ниже утверждения теоремы 1 расширяются и уточняются следующим образом. Достаточное условие $m<n-2$ вполне квазиомбиличности заменяется более слабым условием $m_{1}<n-2$, а метод доказательства, примененный в $\left[{ }^{8}\right]$, - более геометрическим способом доказательства. Для случая $m_{1} \geqslant n-2$ дается геометрическое толкование условия (1). В итоге получена следующая теорема, доказанная в $\$ 2$.

Т еорем а 2. Пусть $V_{n}(n>3)$ является конформно плоским подмногообразием с плоской нормальной связностью в конформно плоском $V_{n+m}$. Если на него $m_{1}<n-2$, то $n-m_{1}$ главных векторов кривизны совпадают между собой $и$ определяют особую вершину прямоугольного симплекса, являющегося индикатрисой нормальной кривизны, а само $V_{n}$ является вполне квазиомбилическим. Если в точке $X \in V_{n}$ имеет место $m_{1} \geqslant n-2$, то либо $m_{1}=n-1$ и индикатриса нормальной кривизны в этой точке является ортоцентрическим симплексом, либо $m_{1}=n-2 u$ эта индикатриса является выпуклой оболочкой орточентрического симплекса и его ортоцентра.

3. Основная часть настоящей статьи посвящена выяснению вопроса, поставленного в $\left[{ }^{7}\right]$, могут ли среди конформно плсских подмногообразий $V_{n}(n>3)$ с плоской нормальной связностью в конформно плоском $V_{n+m}$ существовать такие $V_{n}$, которые не являются вполне квазиомбилическими. Ниже приведены новые примеры, которые позволяют изучить этот вопрос. Они найдены среди изотермических подмногообразий, которые, как оказывается, составляют другой интересный подкласс конформно плоских подмногообразий $V_{n}$ с плоской нормальной связностью в конформно плоском $V_{n+m}$, наряду с вполне квазиомбилическими.

Изометрические гиперповерхности в $E_{n+1}$ были введены в $\left[{ }^{11,12}\right.$ ] как естественные обобщения классических изотермических поверхностей в 
$E_{3}$. Они допускают дальнейшее обобщение в классе подмногообразий с плоской нормальной связностью следующим образом.

Атлас локальных карт на конформно плоском $V_{n}$ называется изотермическим, если в его любой карте

$$
d s^{2}=e^{-2 \sigma}\left(d u_{1}^{2}+\ldots+d u_{n}^{2}\right),
$$

где $\sigma-$ некоторая гладкая функция на области этой карты.

Опред еление. Нзотермическим называется всякое конформно плоское подмногообразие $V_{n}$ с плоской нормальной связностью в конформно плоском пространстве $V_{n+m}$, которое обладает таким изотермическим атласом, что сеть координатных линий каждой карты этого атласа является сетью линий кривизны этого подмногообразия $V_{n}$.

Класс изотермических подмногообразий $V_{n}$ в конформнс плоском $V_{n+m}$ обозначим через $I_{(n, m)}$. Этот класс непустой. Ниже в $\S 3$ найдена система пфаффовых уравнений, определяющая подмногообразие $V_{n} \in$ $\in I_{(n, m)}$ самого общего типа, и доказана ее совместность (предложение 1). Класс изотермических подмногообразий $V_{n}$ в $V_{n+m}$ с попарно различными главными векторами кривизны обозначим через $I_{(n, m)}^{(n)}$.

В этом классе существует непустой подкласс, состоящий из подмногообразий, главные векторы кривизн которых параллельны в нормальной связности вдоль соответствующих им линий кривизны $(\$ 3$, предложение 2). Этот подкласс тесно связан с подмногообразиями, которые обладают следующими интересными геометрическими свойствами.

Подмногообразие $V_{n}$ с плоской нормальной связностью в $E_{n+m}$, все линии кривизны которого окружности (плоскости которых для линий каждого семейства либо имеют общую прямую, либо параллельны между собой), будем называть подмногообразием Дюпена-Маннгейма (в частном случае $m=1$ они были рассмотрены в $\left[{ }^{11,12}\right]$ ).

Подмногообразие $V_{n}$ с плоской нормальной связностью в $E_{n+m}$, все главные векторы кривизны которого параллельны в нормальной связности, называется изопараметрическим (см. $\left.\left[{ }^{13,14}\right]\right)$.

Имеют место следующие теоремы, доказываемые в $\S 3$ и 4 .

Т е о р е а 3. Если $V_{n} \in I_{(n, n-2)}^{(n)}(n \geqslant 3)$, то все его главные векторы кривизны параллельны в нормальной связности вдоль соответствующцих им линий кривизны.

Т е о р е м а 4. Подмногообразие $V_{n}$ класса $\underset{(n, m)}{I_{(n)}^{(n)}}(n \geqslant 3)$, главные векторы кривизны которого параллельны в нормальной связности вдоль соответствующих им линий кривизны, является объединением замыканий своих областей, отображающихся локальными конформными отображениями $V_{n+m}$ в $E_{n+m}$

1) при отличных от нуля главных векторах кривизны: на подмногообразие Дюпена-Маннгейма,

2) при одном главном векторе кривизны, равным нулю:

а) на конус, который проектирует (n-1)-мерную обобщценную поверхность Клиффорда из центра содержащей ее гиперсферы, либо

б) на изопараметрический цилиндр над произведением (n-1) окружностей, т. е. предельный случай предыдущего, когда вериина конуса удаляется в бесконечность.

\section{§ 2. Доказательство теоремы 2}

1. Пусть $V_{n}$ является подмногообразием с плоской нормальной связностью в конформно плоском $V_{n+m}$. Так как сеть его линий кривизны и понятие изотермичности сети конформно инвариантны, то при локальных рассмотрениях, используя известный прием (см. $\left.\left[{ }^{15}\right]\right)$, можно без 
ограничения общности конформно плоское $V_{n+m}$ заменить на евклидово пространство $E_{n+m}$.

Пусть $V_{n}$ является изотермическим подмногообразием в $E_{n+m}$. Оно является базой своего касательного векторного расслоения $T\left(V_{n}\right)$ и нормального векторного расслоения $T \perp\left(V_{n}\right)$. Слоями этих расслоений являются соответственно касательное пространство $T_{X}\left(V_{n}\right)$ и нормальное пространство $T_{X} \perp\left(V_{n}\right)$ как векторные подпространства в $T_{X}\left(E_{n+m}\right)$. Присоединим к любой точке $X \in V_{n}$ ортонормированный подвшжный репер так, чтобы множества векторов $\left\{\mathbf{e}_{1}, \ldots, \mathbf{e}_{n}\right\}$ и $\left\{\mathbf{e}_{n+1}, \ldots, \mathbf{e}_{n+m}\right\}$ были базисами соответственно в $T_{X}\left(V_{n}\right)$ и в $T_{X}^{\perp}\left(V_{n}\right)$, причем векторы первого базиса были касательными к линиям кривизны. В формулах инфинитезимального перемещения репера

$$
d \mathbf{x}=\mathbf{e}_{J} \omega^{J}, \quad d \mathbf{e}_{K}=\mathbf{e}_{J} \omega_{K}^{J} ; \quad J, K=1, \ldots, n, n+1, \ldots, n+m,
$$

имеем $\omega_{J}^{K}=-\omega_{K}^{J}, d \omega^{J}=\omega^{K} \wedge \omega_{K}^{J}, d \omega_{K}^{J}=\omega_{K}^{L} \wedge \omega_{L}^{J}, \quad$ и в данном случае $\omega^{\alpha}=0, \quad \omega_{p}^{\alpha}=k_{p}^{\alpha} \omega^{p} ; \quad p=1, \ldots, n ; \quad \alpha, \beta, \gamma, \ldots=n+1, \ldots, n+m$

(по индексу $p$ не суммировать!). Векторы

$$
\mathbf{k}_{p}=\sum_{\alpha} k_{p}^{\alpha} \mathbf{e}_{\alpha}
$$

называются главными векторами кривизны.

2. Докажем первое утверждение теоремы 2. Пусть, как и раньше,

$$
m_{1}=\operatorname{dim} A_{X}\left(K_{1}, \ldots, K_{n}\right),
$$

где $A_{X}\left(K_{1}, \ldots, K_{n}\right)$ - наименьшее аффинное подпространство в $T_{X}^{\perp}\left(V_{n}\right)$, которое содержит все точки $K_{1}, \ldots, K_{n}$, и пусть $m_{1} \leqslant n-3$. Путем перенумерации главных направлений (т. е. векторов $\mathbf{e}_{1}, \ldots, \mathbf{e}_{n}$ ) можно достичь, чтобы векторы $\mathbf{k}_{1}, \ldots, \mathbf{k}_{m_{1}+1}$ были радиусами-векторами точек $K_{1}, \ldots, K_{m_{1}+1}$ общего положения в $A_{X}\left(K_{1}, \ldots, K_{n}\right)$. В этом случае точки $K_{1}, \ldots, K_{m_{1}+1}$ образуют $m_{1}$-мерный симплекс, который в силу (1) является ортоцентрическим. Этот симплекс обозначим через $\Delta_{X}$. Осталось выяснить, где могут помещаться остальные точки $K_{m_{1}+2}, \ldots, K_{n}$ с радиусами-векторами $\mathbf{k}_{m_{1}+2}, \ldots, \mathbf{k}_{n}$, которых не меньше двух.

Сначала покажем, что одна из них совпадает с некоторой вершиной симплекса $\Delta_{X}$.

Возьмем точку $K_{m_{1}+2}$. Если она совпадает с одной из вершин $K_{1}, \ldots, K_{m_{1}+1}$, то цель достигнута. В противном случае для нее остается лишь одна возможность: она должна быть ортоцентром симплекса $\Delta_{X}$, как следует из (1).

Так как $m_{1}+3 \leqslant n$, то существует следующая точка $K_{m_{1}+3}$. Если она совпадает с одной из вершин, то цель достигнута. В противном случае возникает противоречие. Действительно, в силу (1) для любых различных $K_{i_{1}}$ и $K_{j_{1}}$, являющихся вершинами симплекса $\Delta_{X}$, должно быть

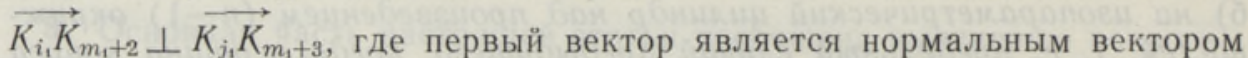
грани симплекса $\Delta_{x}$, не содержащей вершину $K_{i_{1}}$. Это говорит о том, что $K_{m_{1}+3}$ лежит на этой грани симплекса $\Delta_{X}$. Так как это относится к любой грани, то противоречие получено, потому что все грани симплекса $\Delta_{X}$ не могут иметь общую точку.

Перенумеруем вершины так, чтобы $K_{m_{1}+3}$ совпадала с вершиной $K_{m_{1}+1}$. Из (1) следует, что

$\left\langle{\vec{K} m_{1}+1}_{K_{\rho}}, \vec{K}_{m_{1}+{ }_{3} K_{\pi}}\right\rangle=\left\langle{\overrightarrow{K_{m_{1}+1} K_{\rho}}}_{K_{m_{1}+1} K_{\pi}}\right\rangle=0, \quad\left(\varrho, \pi=1, \ldots, m_{1} ; \varrho \neq \pi\right)$, т. е. ребра, которые выходят из вершины $K_{m_{1}+1}=K_{m_{1}+3}$, ортогональны 
ӥ их веќторы можнно ис́пользовать в ка̀честве б́азиса в $m_{1}$-мерном $A_{X}\left(K_{1}, \ldots, K_{n}\right)$. Следовательно,

$$
\vec{K}_{m_{1}+3} K_{m_{1}+2}=\sum_{\rho} \xi_{m_{1}+2}^{\rho} \vec{K}_{m_{1}+1} K_{\rho}, \ldots, \vec{K}_{m_{1}+3} K_{n}=\sum_{\rho} \xi_{n}^{\rho} \vec{K}_{m_{1}+1} K_{\rho},
$$

а из (1) следует, что написанные здесь векторы равны нулю, т. е. $K_{m_{1}+1}=K_{m_{1}+2}=\ldots=K_{n}=K$. Этим при $m_{1} \leqslant n-3$ доказано утверждение, касающееся индикатрисы нормальной кривизны. Остается показать, что $V_{n}$ вполне квазиомбилическое.

Обозначим радиус-вектор особой вершины $K$ через k. Репер в нормальном пространстве $T_{X} \perp\left(V_{n}\right)$ специализируем так, что $\mathbf{e}_{n+\rho} \| \overrightarrow{K K}_{\rho}\left(\varrho=1, \ldots, m_{1}\right)$; векторы $\mathbf{e}_{n+m_{1}+1}, \ldots, \mathbf{e}_{n-m}$ идут тогда в ортогональное дополнение к $A_{X}\left(K_{1}, \ldots, K_{n}\right)$ в $T_{X} \perp\left(V_{n}\right)$. Матрица второй фундаментальной формы в направлении $\mathbf{e}_{n+\rho}$ имеет при этом вид

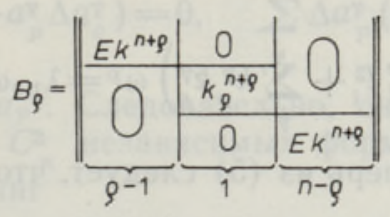

где $k^{n+\rho}=\left\langle\mathbf{k}, \mathbf{e}_{n+\rho}\right\rangle, k_{\rho}^{n+\rho}=\left\langle\mathbf{k}_{\rho}, \mathbf{e}_{n+\rho}\right\rangle$, а $E$ является единичной матрицей нужного порядка. В направлении вектора $\mathbf{e}_{n+\lambda}\left(\lambda=m_{1}+1, \ldots\right.$ $\ldots, m)$ имеем $B_{\lambda}=k^{n+\lambda} E$, где $k^{n+\lambda}=\left\langle\mathbf{k}, \mathbf{e}_{n+\lambda}\right\rangle$. Следовательно, $V_{n}$ является вполне квазиомбилическим. Первое утверждение теоремы 2 доказано.

3. Докажем второе утверждение этой теоремы, Пусть $m_{1}=\operatorname{dim} A_{X}\left(K_{1}, \ldots, K_{n}\right) \geqslant n-2>1$. Точки $K_{1}, \ldots, K_{m_{1}+1}$ с радиус-векторами $\mathbf{k}_{1}, \ldots, \mathbf{k}_{m_{1}+1}$ образуют симплекс $\Delta_{X}$ в $A_{X}\left(K_{1}, \ldots, K_{n}\right)$. Из (1) следует, что симплекс $\Delta_{X}$ является ортоцентрическим, так как его неприлежащие ребра ортогональны.

Так как всегда $m_{1} \leqslant n-1$, то при $m_{1} \geqslant n-2$ возможны лишь два случая: либо $m_{1}=n-1$, либо $m_{1}=n-2$. В первом случае радиус-векторы вершин симплекса $\Delta_{X}$ исчерпывают все главные векторы кривизны. Во втором случае остается еще вектор $\mathbf{k}_{m_{1}+2}=\mathbf{k}_{n}$, который в силу (1) является радиус-вектором ортоцентра симплекса $\Delta_{X}$. Теорема доказана.

3 а ме ч н и е 1. При $m_{1}=n-2$ может оказаться, что ортоцентр совпадает с одной из вершин симплекса $\Delta_{x}$, которая является тогда особой вершиной прямоугольного симплекса. Также как в предыдущем пункте можно показать, что если это имеет место в любой точке $V_{n}$, то $V_{n}$ является вполне квазиомбилическим.

Отметим также, что если ортоцентр находится внутри симплекса $\Delta_{\boldsymbol{X}}$, то индикатриса нормальной кривизны совпадает с $\Delta_{\boldsymbol{X}}$.

3 а м еч а и е 2. Если подмногообразие $V_{n}$ в ситуации теоремы 2 имеет попарно различные главные векторы кривизны в каждой своей точке, то либо $m_{1}=n-1$, либо $m_{1}=n-2$ и индикатриса нормальной кривизны не является прямоугольным симплексом.

\section{§3. Доказательство теоремы 3}

1. Подготовим сперва нужный аналитический аппарат. Пусть дано подмногообразие $V_{n} \in I_{(n, m)}$. Из (2) следует, что для его изотермического атласа $\omega^{p}=\mathrm{e}^{-\sigma} d u_{p}$ и поэтому $d\left(e^{\sigma} \omega^{p}\right)=0$, т. е. 


$$
d \sigma \wedge \omega^{p}+\sum_{q=1}^{n} \omega^{q} \wedge \omega_{q}^{p}=0
$$

Отсюда $d \sigma=\sum_{q} l_{q} \omega^{q}, \omega_{q}^{p}+l_{q} \omega^{p}=\sum_{r \neq p} \lambda_{q r}^{p} \omega^{r}$, где $\lambda_{q r}^{p}=\lambda_{r q}^{p}(p \neq q, p \neq r)$. Если учесть здесь $\quad \omega_{p}^{q}+\omega_{q}^{p}=0, \quad$ получим $\quad \lambda_{q q}^{p}=l_{p}, \lambda_{q r}^{p}+\lambda_{p r}^{q}=0$ при различных $p, q, r$. Последнее равенство дает, что $\lambda_{p r}^{q}=0$ при $p \neq q \neq r \neq p$. Следовательно,

$$
\omega_{q}^{p}=l_{p} \omega^{q}-l_{q} \omega^{p} .
$$

Из (4) получается дифференциальным продолжением, что

$$
d k_{p}^{\alpha}=a_{p}^{\alpha} \omega^{p}+\sum_{q \neq p} l_{q}\left(k_{p}^{\alpha}-k_{q}^{\alpha}\right) \omega^{q}-\sum_{\nu} k_{p}^{\gamma} \omega_{\nu}^{\alpha}
$$

а из (6), что

$$
d l_{p}-\frac{1}{2}\left(\sum_{i} l_{i}^{2}+\sum_{\gamma} k_{p}^{v} k_{q}^{\gamma}\right) \omega^{p}=\lambda_{p q} \omega^{q}+\mu_{p q} \omega^{p},
$$

где $\mu_{p q}=-\mu_{q p}, q \neq p$. Теперь из (5) следует, что

$$
d \mathbf{k}_{p}=\left(\mathbf{a}_{p}-\left\langle\mathbf{k}_{p}, \mathbf{k}_{p}\right\rangle \mathbf{e}_{p}\right) \omega^{p}+\sum_{q \neq p}\left[l_{q}\left(\mathbf{k}_{p}-\mathbf{k}_{q}\right)-\left\langle\mathbf{k}_{p}, \mathbf{k}_{q}\right\rangle \mathbf{e}_{q}\right] \omega^{q},
$$

где $\mathbf{a}_{p}=\sum_{\gamma} a_{p} \mathbf{e}_{\gamma}$

Предположим, что $n \geqslant 3$. Заменим в (8) индексное значение $q$ значением $r, r \neq q, r \neq p$. Получаются равенства $\lambda_{p q}=\lambda_{p r}=0 \quad$ и $\mu_{p r}-\mu_{p q}=\frac{1}{2}\left\langle\mathbf{k}_{p}, \mathbf{k}_{q}-\mathbf{k}_{r}\right\rangle$. Если произвести циклирование по $p, q, r$ и учесть $\mu_{p q}+\mu_{q p}=0$, то в результате имеем $\mu_{p q}=\frac{1}{2}\left\langle\mathbf{k}_{r}, \mathbf{k}_{p}-\mathbf{k}_{q}\right\rangle$. Подстановка в (8) дает

$$
d l_{p}=\frac{1}{2}\left(\sum_{i} l_{i}^{2}+\left\langle\mathbf{k}_{p}, \mathbf{k}_{q}+\mathbf{k}_{r}\right\rangle-\left\langle\mathbf{k}_{q}, \mathbf{k}_{r}\right\rangle\right) \omega^{p} .
$$

Если $n \geqslant 4$, то из (10) следует, что

$$
\left\langle\mathbf{k}_{p}-\mathbf{k}_{q}, \mathbf{k}_{r}-\mathbf{k}_{s}\right\rangle=0
$$

при четырех различных значениях $p, q, r, s$. Заметим, что (11) согласуется с утверждением теоремы 1, так как изотермическое подмногообразие по своему определению конформно плоское.

Из (10) дифференциальным продолжением получастся конечное соотношение

$$
\left\langle\mathbf{a}_{p}, \mathbf{k}_{q}-\mathbf{k}_{r}\right\rangle=0,
$$

а из (7) система ковариантов

$$
\Delta a_{p}^{\alpha} \wedge \omega^{p}=0
$$

где $\Delta a_{p}^{\alpha}=d a_{p}^{\alpha}+\sum_{v} a_{p}^{\gamma} \omega_{\gamma}^{\alpha}-2 a_{p}^{\alpha} \sum_{i \neq p} l_{i} \omega^{i} . \quad$ Из (12) путем дифференцирования получаются $C_{n}^{2}$ линейных соотношений

$$
\left\langle\mathbf{a}_{p}, \mathbf{a}_{q}\right\rangle=0 .
$$

Предложени е 1. Самое общее подмногообразие $V_{n}$ класса 
$\dot{I}_{(n, m)}(n>3)$ определяется в каноническом репере системой, которая соेстоит из уравнений (4), (6), (7), (10) при конечных соотношениях (11), (12), (14). Эта система находится в инволюции; следовательно; класс $I_{(n, m)}$ оказывается непустым.

Доказательст во. Первая часть предложения суммирует предыдущие рассуждения. Осталось убедиться в совместности этой системы в самом общем случае. Применяем метод продолжения системы до инволюции по критерию Картана (см. $\left.\left[{ }^{16}\right]\right)$.

Общий $n$-мерный интегральный элемент системы ковариантов (13) определяется уравнениями

$$
d a_{p}^{\alpha}=A_{p}^{\alpha} \omega^{p}+2 a_{p}^{\alpha} \sum_{i \neq p} l_{i} \omega^{i}-\sum_{\gamma} a_{p}^{\nu} \omega_{\gamma}^{\alpha}
$$

где $A_{p}{ }^{\alpha}$ являются произвольными параметрами.

Из (12) и (14) получаем $C_{n}^{2}+n C_{n-1}^{2}$ содержит линейных соотношений

$$
\sum_{\nu}\left(\Delta a_{p}^{\gamma} a_{q}^{\gamma}+a{ }_{p}^{\gamma} \Delta a_{q}^{\gamma}\right)=0, \quad \sum_{\gamma} \Delta a_{p}^{\gamma}\left(k_{q}^{\gamma}-k_{r}^{\gamma}\right)=0
$$

на вторичные формы $\Delta a_{p}$. Следовательно, система ковариантов (13) содержит $n m-n C_{n-1}^{2}-C_{n}^{2}$ независимых форм $\Delta a_{p}^{v}$ и матрица ее полярной системы имеет ранг

$$
s_{1}=n m-n C_{n-1}^{2}-C_{n}^{2}
$$

поэтому $s_{2}=\ldots=s_{n}=0$.

Параметры $A_{p}{ }^{\alpha}$, как следует из (12) и (14), связаны с $n C_{n-1}^{2}+C_{n}^{2}$ линейными соотношениями вида

$$
\left\langle\mathbf{A}_{p}, \mathbf{k}_{q}-\mathbf{k}_{r}\right\rangle=0, \quad\left\langle\mathbf{A}_{p}, \mathbf{a}_{q}\right\rangle=0,
$$

где $\mathbf{A}_{p}=\sum_{v} A_{p}^{\gamma} \mathbf{e}_{\gamma}$. Так как число произвольных параметров в интегральном элементе равно числу Картана, то рассматриваемая система находится в инволюции.

2. Доказательство теоремы 3 . Пусть $V_{n}$ является подмногообразием класса $I_{(n, n-2)}^{(n)}$. Из замечания 2 и очевидного неравенства $m_{1} \leqslant m=n-2$ следует, что $m_{1}=m=n-2$, т. е. $A_{X}\left(K_{1}, \ldots, K_{n}\right)=$ $=T_{X} \perp\left(V_{n}\right)$ и в этом $(n-2)$-мерном пространстве помещаются $n$ точек $K_{1}, \ldots, K_{n}$, являющихся вершинами ортоцентрического симплекса и его ортоцентром. При любой фиксации $p$ и $q, p \neq q$, найдутся $\mathbf{k}_{q}-\mathbf{k}_{r_{1}}, \ldots$ $\ldots, \mathbf{k}_{q}-\mathbf{k}_{r_{m}}$, составляющие базис в $T_{X}^{\perp}\left(V_{n}\right)$. Так как $\mathbf{a}_{p} \in T_{X} \perp\left(V_{n}\right)$, то из (12) следует, что $\mathbf{a}_{p}=0(p=1, \ldots, n)$. Это означает, как следует из (9), что все главные векторы кривизны являются параллельными в нормальной связности вдоль соответствующих им линий кривизны. Теорема доказана.

Вообще, вопрос о существовании подмногообразия $V_{n} \in \underset{(n, m)}{I_{(n)}}$, главные кривизны которого параллельны в нормальной связности вдоль соответствующих им линий кривизны, решается следующим предложением, которое следует непосредственно из доказательства предложения 1 .

Предложение 2. Подмногообразие $V_{n}$ класса $\underset{(n, m)}{(n)}(n-2 \leqslant$ $\left.\leqslant m_{1} \leqslant m\right)$ при $n=3$ ( $\left.n>3\right)$, главные векторы кривизны которого параллельны в нормальной связности вдоль соответствующцх им линий кривизны, определяется в каноническом репере вполне интегрируемой системой, которая состоит из уравнений (4), (6), (7) u (10), где все $\mathbf{a}_{p}=0$ (при конечных соотношениях (11)). 


\section{§ 3. Доказательство теоремы 4}

1. Начнем с утверждения 1) теоремы 4. Пусть $V_{n}(n \geqslant 3)$ является изотермическим подмногообразием в $E_{n+m}$ с $n$ попарно различными главными векторами кривизны, параллельными в нормальной связности вдоль соответствующих им линий кривизны.

Из (9) следует, что такое подмногообразие $V_{n}$ выделяется условиями $\mathrm{a}_{p}=0 ; p=1, \ldots, n$. Поэтому $V_{n}$ помещается в своей первой соприкасающейся плоскости, определяемой как аффинная оболочка точки $X \in V_{n}$ пространства $T_{X}\left(V_{n}\right)$ и всех главных векторов кривизны $\mathbf{k}_{1}, \ldots, \mathbf{k}_{n}$.

Система, указанная в предложении 2, теперь вполне интегрируема, и рассматриваемая $V_{n}$ существует с произволом постоянных. Ее линии кривизны, являющиеся интегральными линиями систем $\omega^{1}=\ldots=$ $=\omega^{p-1}=\omega^{p+1}=\ldots=\omega^{n}=0$, суть окружности, так как

$$
\begin{gathered}
d \mathbf{e}_{p}=\left(\sum_{i \neq p} l_{i} \mathbf{e}_{i}+\mathbf{k}_{p}\right) \omega^{p}, \\
d\left(\sum_{i \neq p} l_{i} \mathbf{e}_{i}+\mathbf{k}_{p}\right)=-\left(\sum_{i \neq p} l_{i}^{2}+\left\langle\mathbf{k}_{p}, \mathbf{k}_{p}\right\rangle\right) \omega^{p} \mathbf{e}_{p} .
\end{gathered}
$$

Плоскости этих окружностей при заданном $p$ проходят через фиксированную прямую или параллельны между собой.

Действительно, если $l_{p} \neq 0$, то прямая, которая проходит через точку $C_{p}$ с радиус-вектором $\mathbf{c}_{p}=\mathbf{x}+l_{p}^{-1} \mathbf{e}_{p}$ в направлении вектора

$$
\mathbf{m}_{p}=2 l_{p}\left(\sum_{i \neq p} l_{i} \mathbf{e}_{i}+\mathbf{k}_{p}\right)+\left(l_{p}^{2}-\sum_{i \neq p} l_{i}^{2}-\left\langle\mathbf{k}_{p}, \mathbf{k}_{q}+\mathbf{k}_{r}\right\rangle+\left\langle\mathbf{k}_{q}, \mathbf{k}_{r}\right\rangle\right) \mathbf{e}_{p}
$$

где $p, q, r$ - три различных значения индекса $i=1, \ldots, n$, принадлежит плоскости рассматриваемой окружности кривизны и является неподвижным, так как

$$
\begin{gathered}
d\left(\mathbf{x}+l_{p}^{-1} \mathbf{e}_{p}\right)=\frac{1}{2} l_{p}^{-2} \mathbf{m}_{p} \omega^{p}, \\
d \mathbf{m}_{p}=\mathbf{m}_{p} \sum_{i} l_{i} \omega^{i}
\end{gathered}
$$

(заметим, что при $n>3$ в силу (11) выражение вектора (15) не зависит от того, какие значения принимают $q$ и $r$.) Непосредственно проверяется, что $\mathbf{m}_{p} \perp \mathbf{m}_{q}$, т. е. направления этих прямых попарно ортогональны.

Если $l_{p}=0$, то плоскости рассматриваемых окружностей сохраняют свое 2-направление, потому что тогда при любом смещении точки $X$ на рассматриваемом подмногообразии $V_{n}$ имеем

$$
\begin{gathered}
d \mathbf{e}_{p}=\left(\sum_{i \neq p} l_{i} \mathbf{e}_{i}+\mathbf{k}_{p}\right) \omega^{p}, \\
d\left(\sum_{i \neq p} l_{i} \mathbf{e}_{i}+\mathbf{k}_{p}\right)=-\left(\sum_{i \neq p} l_{i}^{2}+\left\langle\mathbf{k}_{p}, \mathbf{k}_{p}\right\rangle\right) \omega^{p} \mathbf{e}_{p}+\sum_{i \neq p} l_{i} \omega^{i}\left(\sum_{j \neq p} l_{j} \mathbf{e}_{j}+\mathbf{k}_{p}\right),
\end{gathered}
$$

так как из (10) следует теперь, что $\sum_{i} l_{i}^{2}+\left\langle\mathbf{k}_{p}, \mathbf{k}_{q}+\mathbf{k}_{r}\right\rangle-\left\langle\mathbf{k}_{q}, \mathbf{k}_{r}\right\rangle=0$ Утверждение 1) доказано.

2. Докажем утверждение 2) теоремы 4. Пусть один вектор главной 
кривизны равен нулю, например, $\mathbf{k}_{n}=0$. Из (9), где $\mathbf{a}_{i}=0$, следует, что $l_{p}=0(p=1, \ldots, n-1)$ и

$$
d \mathbf{k}_{p}=-\left\langle\mathbf{k}_{p}, \mathbf{k}_{p}\right\rangle \mathbf{e}_{p} \omega^{p}+l_{n} \mathbf{k}_{p} \omega^{n}-\sum_{\substack{i=1 \\ i \neq p}}^{n-1}\left\langle\mathbf{k}_{p}, \mathbf{k}_{i}\right\rangle \mathbf{e}_{i} \omega^{i},
$$

а из (10) получается $\left\langle\mathrm{k}_{p}, \mathbf{k}_{q}\right\rangle=-l_{n}^{2}, 1 \leqslant p, q \leqslant n-1, p \neq q$ и

$$
d l_{n}=l_{n}^{2} \omega^{n} .
$$

Теперь 2-направление, натянутое на векторы $\mathbf{e}_{p}$ и $l_{n} \mathbf{e}_{n}+\mathbf{k}_{p}$, инвариантно связано с подмногообразием при каждом значении $p=1, \ldots$ $\ldots, n-1$, так как

$$
\begin{gathered}
d \mathbf{e}_{p}=\left(l_{n} \mathbf{e}_{n}+\mathbf{k}_{p}\right) \omega^{p}, \\
d\left(l_{n} \mathbf{e}_{n}+\mathbf{k}_{p}\right)=l_{n}\left(l_{n} \mathbf{e}_{n}+\mathbf{k}_{p}\right) \omega^{n}-\left(l_{n}^{2}+\left\langle\mathbf{k}_{p}, \mathbf{k}_{p}\right\rangle\right) \omega^{p} \mathbf{e}_{p} .
\end{gathered}
$$

Пусть $l_{n} \neq 0$. Плоскость, которая проходит через точку $Y$ с радиусвектором $\mathbf{y}=\mathbf{x}+l_{n}{ }^{-1} \mathbf{e}_{n}$ с этим инвариантным 2-направлением, неподвижна, так как $d \mathbf{y}=0$ при любом смещении вдоль подмногообразия $V_{n}$. Линии кривизны в направлении вектора е ${ }_{n}$ являются прямыми, которые проходят через неподвижную точку $Y$ и образуют постоянный угол $\alpha_{p}$ каждой из вышеуказанных неподвижных 2-плоскостей. Действительно, проекция этой прямой на рассмотренной неподвижной 2-плоскости имеет направляющий вектор $l_{n} \mathbf{e}_{n}+\mathbf{k}_{p}$ и $d\left(\cos ^{2} \alpha_{p}\right)=0$.

В терминах эллиптической геометрии подмногообразие кривизны, вдоль которой $\omega^{n}=0$ является $(n-1)$-мерной обобщенной поверхностью Клиффорда $\left[{ }^{17}\right]$ и $V_{n}$ является конусом над ней.

Пусть $l_{n}=0$. Ненулевые главные векторы кривизны являются попарно ортогональными и $m \geqslant n-1$. Очевидно, такое подмногообразие является изопараметрическим. Оно устроено как цилиндр над произведением $(n-1)$-окружностей. Действительно, прямые на $V_{n}$ с направляющим вектором $\mathbf{e}_{n}$ являются параллельными, а их ортогональное сечение, выделяемое уравнением $\omega^{n}=0$ является произведением окружностей на плоскостях пар векторов $\left(\mathbf{e}_{p}, \mathbf{k}_{p}\right)$. Теорема 4 доказана.

\section{ЛИ Т Е Р А Т У РА}

1. Cartan, E. Bull. Soc. math. France, 45, 57-121 (1917).

2. Schouten, J. A. Math. Z., 11, 58-88 (1921).

3. Chen, B.-Y. Geometry of Submanifolds. New York, M. Dekker, 1973.

4. Вербицкий Л. Л. Тр. семин. по вект. и тензорн. анал., вып. 9, 146-182 (1952).

5. Gebarowski, A. Demonstr. Math., 6, № 2, 641-646 (1973).

6. Amur, K., Pujar, S. Tensor (N. S.), 32, 62-64 (1978).

7. Chen, B.-Y., Teng, T. H., Soochow, J. Math. Natur. Sci., 1, № 1, 9-16 (1975).

8. Chen, B.-Y., Verstraelen, L. Boll. Unione mat. ital., A14, № 1, 49-57 (1977).

9. Схоутен И. А., Стройк Д. Дж. Введение в новые методы в дифференциальной геометрин. М., Гос. изд. ин. лит., 1948.

10. Крейцер Г. П., Тюрин Г. И. Матем. просвещение, вып. 2, 188-194 (1957).

11. Лумисте Ю. Уч. зап. Тартуск. ун-та, 734, 36-49 (1986).

12. Вяльяс М. Уч. зап. Тартуск. ун-та, $734,20-30$ (1986).

13. Harle, C. E. Boll. Soc. Bras. Mat., 13, № 2, 35-48 (1982).

14. Strübing, W. Geom. dedic,, 20, № 3, 367-387 (1986).

15. Chen, B. $-Y$. Boll. Unione mat. ital., 10, 380-385 (1974).

16. Фиников С. П. Метод внешних форм Картана в дифференциальной геометрии. М.-Л., Гостехнздат, 1948.

17. Розенфельд Б. А. Неевклидовы пространства. М., «Наука», 1969.

Тартуский государственный университет
Поступила в редакцию 9/I 1987 


\section{ISOTERMILISTEST ALAMMUUTKONDADEST KONFORMSELT TASASES RUUMIS}

Töös on antud alammuutkonna normaalkõveruse indikatrissi mõistet kasutades geomeeiriline tôlgendus seosele $\left(^{*}\right)$, on defineeritud isotermilised alammuutkonnad ja tõestatud nende olemasolu. Isotermiliste alammuutkondade hulgas on leitud näited konformselt tasastest alammuutkondadest, mis pole täielikult kvaasiombilised.

\section{VÄLJAS}

\section{ON ISOTHERMAL SUBMANIFOLDS IN A CONFORMALLY FLAT SPACE}

It is known that a hypersurface $V_{n}(n>3)$ in a conformally flat space $V_{n+1}$ is conformally flat if and only if it is quasiumbilical $\left[{ }^{1,2}{ }^{2}\right]$. In $\left[{ }^{8}\right]$ this result was generalized and the next condition on principal curvature vectors $\mathbf{k}_{1}, \ldots, \mathbf{k}_{n}$ of $V_{n}$ with flat normal connection $\nabla^{\perp}$ was found

$$
\left\langle\mathbf{k}_{p}-\mathbf{k}_{q}, \mathbf{k}_{r}-\mathbf{k}_{s}\right\rangle=0
$$

with mutually different $p, q, r, s$. This is necessary and sufficient for $V_{n}$ to be conformally ilat. As a consequence, a $V_{n}$ with flat $\nabla^{\perp}$ is totally quasiumbilical always if $m<n-2$.

The normal curvature vector in the direction of unit tangent vector $\mathbf{f}=\sum_{i=1}^{n} \varphi^{i} \mathbf{e}_{i}$, where $\mathrm{e}_{1}, \ldots, \mathrm{e}_{n}$ are unit tangent vectors of curvature lines, is $\mathbf{k}=\sum_{i=1}^{n} \mathbf{k}_{i}\left(\varphi^{i}\right)^{2}$. At each point $X \in V_{n}$ the next set of points $\{K: \overrightarrow{X K}=\mathbf{k}\}$ is the normal curvature (indicatrix (see [ $\left.\left.{ }^{9}\right]\right)$; let $m_{1}$ be the dimension of its affine hull. If $\operatorname{dim} T_{X}^{\perp}\left(V_{n}\right) \geqslant n-1$ and the points $K_{1}, \ldots, K_{n}$ are in a general position, where $\overrightarrow{X K}_{i}=\mathbf{k}_{i}$, the normal curvature indicatrix is a simplex.

In the first part of this paper, a geometrical interpretation of the condition $(*)$ is given, and a generalization of the consequence is proved by a new method.

The orem 2. Let $V_{n} \quad(n>3)$ be a submanifold with flat normal connection in a conformally flat space $V_{n+m}$. If $m_{1}<n-2$, then $V_{n}$ is totally quasiumbilical. If $m_{1} \geqslant$ $\leqslant n-2$ at a point $X \in V_{n}$, then normal curvature indicatrix at $X$ is convex hull of an orthocentral simplex and its orthocentre (or the orthocentral simplex itself, if the latter contains its orthocentre).

In the second part of this paper, new examples of conformally flat submanifolds $V_{n}$ with flat normal connection are given. They are found among isothermal submanifolds and, in general, are not totally quasiumbilical.

Definition. A conformally flat submanifold $V_{n}$ with flat normal connection in a conformally flat space $V_{n+m}$ is called isothermal, if it has an isothermal atlas whose net of coordinate lines in each card is a net of curvature lines.

Submanifo!d $V_{n}$ with flat normal connection in $E_{n+m}$ is called a Dupin-Manngheim submanifold, if its families of curvature lines consist of circles whose planes have a common straight line or are parallel to each other.

The or e m 3et $V_{n}(n \geqslant 3)$ be an isothermal submanifold in a conformally flat space $V_{2(n-1)}$ with $n$ mutually different principal curvature vectors. Then these vectors are parallel in normal connection along their corresponding curvature lines.

The o r em 4. An isothermal submanifold $V_{n}(n \geqslant 3)$ in a conformally flat $V_{n+m}$ $\left(n-2 \leqslant m_{1} \leqslant m\right)$ with $n$ mutually different principal curvature vectors, parallel in normal connections along their corresponding curvature lines, is a union of closures of donains, whose images under local conformal mappings from $V_{n+m}$ in to $E_{n+m}$ are

$1_{1}$ in case of non-zero principal curvature vectors: submanifolds of DupinMan igheim.

2) in case of one zero principal curvature vector: a) Clifford cones (projecting $(n-1)$-dimensional Clifford submanifolds from centres of hyperspheres containing them) or b) isoparametric cylinders on products of $(n-1)$ circles (i.e. limit cases of previous cones if the vertices tend to infinity). 